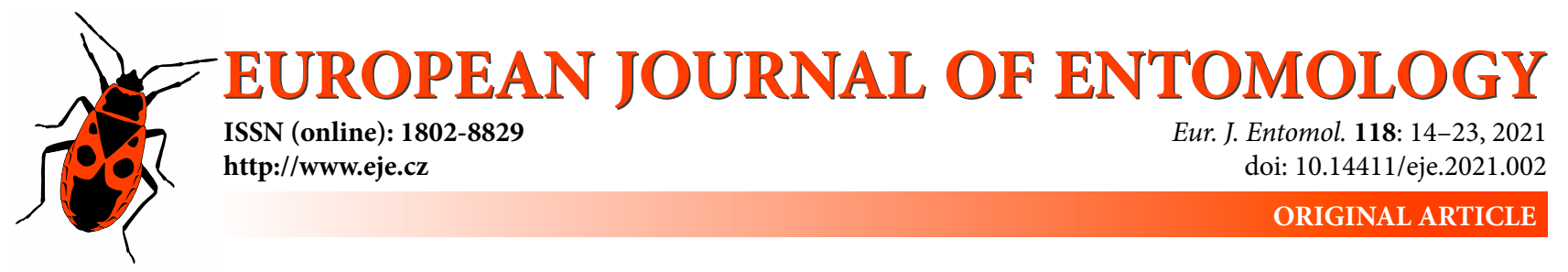

\title{
The associations between ground beetle (Coleoptera: Carabidae) communities and environmental condition in floodplain forests in the Pannonian Basin
}

\author{
Juraj LITAVSKÝ ${ }^{1}$, Oto MAJZLAN ${ }^{1}$, SLAVomí STAŠIOV ${ }^{2}$, MAREK SVITOK $^{2,3}$ and Peter FEDOR ${ }^{1}$ \\ ${ }^{1}$ Department of Environmental Ecology and Landscape Management, Faculty of Natural Sciences, Comenius University, \\ Ilkovičova 6, SK-842 15 Bratislava, Slovakia; e-mails: litavskyjuraj@gmail.com, oto.majzlan@uniba.sk, peter.fedor@uniba.sk \\ ${ }^{2}$ Department of Biology and General Ecology, Faculty of Ecology and Environmental Sciences, Technical University in Zvolen, \\ T.G. Masaryka 24, SK-960 53 Zvolen, Slovakia; e-mails: stasiov@tuzvo.sk, svitok@tuzvo.sk \\ ${ }^{3}$ Department of Ecosystem Biology, Faculty of Science, University of South Bohemia, Branišovská 1760, 37005 České \\ Budějovice, Czech Republic
}

Key words. Coleoptera, Carabidae, floodplain forests, Slovakia, Serbia, environmental associations, bioindicators

\begin{abstract}
We studied assemblages of carabids in eight similar habitats, five in Slovakia and three in Serbia. The ground beetles were caught by pitfall traps from February 2015 until November 2016. We compared the incidence of Carabidae in floodplain forests and ecotones alongside the River Danube in Slovakia and the Rivers Tisza and Begej in Serbia. We determined their association with anthropogenic effects, diversity of plants in the different vegetation layers, cover of vegetation layers (herbaceous plants, shrubs and trees), area of forest stands, circumference of forest stands, distance to forest edge, age of forest stands, depth of leaf litter and physico-chemical properties of soil and leaf litter (conductivity, pH, relative content of H, C, P and N). In total, 2,495 adult individuals of 110 species of carabids were collected. The total epigeic activity of the carabids was significantly and positively associated with the number of species of plants in $\mathrm{E}_{3}$ vegetation layer and the relative content of $\mathrm{N}$, and negatively with the cover of the $E_{1}$ layer. Species richness was significantly positively associated with the number of species of plants in the $E_{3}$ layer and the $\mathrm{pH}$ of leaf litter, but an opposite trend in evenness.
\end{abstract}

\section{INTRODUCTION}

Currently, there are very few areas of floodplain forests left in Europe. The vast majority have been destroyed and many of the remaining fragments are in a poor condition. According to Ábrahámová et al. (2014), they are among the most endangered natural ecosystems in Europe. Therefore, it is important to record the current conditions in these habitats over the widest possible area using important bioindicators such as Carabidae. This was the main reason for studying the alluvial soils of three rivers with analogous habitat characteristics, situated at the same latitude and approximately $400 \mathrm{~km}$ apart.

Carabidae are a taxonomically stable and well studied family, which because of their specific life strategies and ecological preferences in terms of humidity, temperature, shading, soil and vegetation (Migliorini et al., 2002; Rainio \& Niemelä, 2003; Boháč, 2005; Schwerk, 2008; Vician et al., 2018) are frequently used for monitoring habitats. As the third largest Coleopteran family (Bouchard et al., 2017), with more than 40,000 species globally (Erwin, 1991; Lövei \& Sunderland, 1996), carabids occur worldwide, from Arctic and alpine tundras to coastal areas, deserts and rainforests (Lövei \& Sunderland, 1996). In various countries in different parts of the Pannonian Basin (Central Europe), ground beetles are abundant, with 619 species recorded in Serbia (Ćurčić et al., 2007, 2018; Guéorguiev, 2008; Hlaváč \& Magrini, 2016; Pavićević et al., 2018), 590 in Slovakia (Zahradník, 2017; Jászay \& Jászayová, 2019) and 517 in Hungary (Horvatovich, 1993; Ádám, 1996).

Various insects, including beetles, are widely used to indicate specific habitat characteristics (Bishop et al., 2009), including environmental disturbance (Niemelä et al., 2000; Pearson \& Cassola, 2005, 2007; Kaiser et al., 2009; Song et al., 2009; Negro et al., 2010; Vásquez-Vélez et al., 2010; Štefánik \& Fedor, 2020), effects of management (Rushton et al., 1990; Jacobs et al., 2010; Kotze et al., 2011; Skłodowski, 2014; Ivanič Porhajašová et al., 2019), restoration (Rothenbücher \& Schaefer, 2006; Babin-Fenske \& Anand, 2010; Paoletti et al., 2010; Liu et al., 2013), forest fragmentation (Niemelä et al., 1988; Niemelä, 2001; Dubovský et al., 2010; Zvaríková et al., 2016) or effects on agricultural ecosystems (Basedow, 1990; Kromp, 1999; Kagawa \& Maeto, 2014; Ivanič Porhajašová et al., 2016). Some species of carabids are sensitive indicators of pollu- 
tion (García et al., 2010; Ito et al., 2010) and are used in studies on urban ecology (Eyre \& Luff, 1990; Venn, 2003) and in ecological assessment.

A number of endangered ground beetles, particularly species of Abax Bonelli, 1810, Badister Clairville, 1806, Calosoma Weber, 1801, Carabus Linnaeus, 1758, Harpalus Latreille, 1802, Leistus Frölich, 1799, Platynus Bonelli, 1810, Pterostichus Bonelli, 1810) and Trechus Clairville, 1806 are associated with natural forests (Boháč, 2005), which is an important factor when considering their conservation. There are a lot of studies on ground beetles in floodplain forests (Šustek, 1994a, c; Antvogel \& Bonn, 2001; Bonn \& Schröder, 2001; Günther \& Assmann, 2005; De Vaate et al., 2007; Porhajašová et al., 2010; Porhajašová \& Šustek, 2011; Majzlan \& Litavský, 2015, 2017; Paill et al., 2018), however, there are no comparisons of the situations in different countries and floodplains, or of their specific ecological variables (area of forest, age of trees, structure of vegetation, anthropogenic effects, content of nutrients in soil, $\mathrm{pH}$, etc.).

The aim of this study is to determine the specificities of carabid communities in alluvial forests and the main ecological and environmental factors associated with their diversity and dynamics. For these reasons, we compared similar types of habitats on the alluvial soils of three rivers (the Danube in Slovakia and the Tisza and the Begej in Serbia) in different geographical regions, by analysing environmental parameters, such as anthropogenic effect, plant species diversity and cover of herbaceous plant layer $\left(E_{1}\right)$, shrub layer $\left(E_{2}\right)$ and tree layer $\left(E_{3}\right)$, size of area, edge of area, circumference of area, age of trees, depth of leaf litter, $\mathrm{pH}$ of the soil and leaf litter, conductivity, content of $\mathrm{P}, \mathrm{N}, \mathrm{C}, \mathrm{H}$ in the soil and leaf litter and their association with particular ground beetle communities.

\section{MATERIAL AND METHODS}

\section{Sites studied}

This research was conducted at eight sites located on the alluvial soils of three rivers: the Danube in Slovakia and the Begej and the Tisza in Serbia. Of these, five sites (S1-S5) are situated on the outskirts of Bratislava in Slovakia (Fig. 1). The mean annual temperature in the area of Bratislava (from 1991 to 2015) was $10.2^{\circ} \mathrm{C}$, average annual relative humidity $72.2 \%$ and mean annual total precipitation $676.2 \mathrm{~mm}$ (Lapin et al., 2019).

S1-located near the overpass at Bajkalská Street $\left(48^{\circ} 8^{\prime} 22.31^{\prime \prime} \mathrm{N}\right.$, $17^{\circ} 8^{\prime} 57.03^{\prime \prime} \mathrm{E}, 138 \mathrm{~m}$ a.s.1.) is a fragment of floodplain forest of the Salici-Populetum association, with a semi-open habitat and a slightly moist microclimate.

S2 - located in the settlement Malé Pálenisko $\left(48^{\circ} 8^{\prime} 11.54^{\prime \prime} \mathrm{N}\right.$, $17^{\circ} 9^{\prime} 14.45^{\prime \prime} \mathrm{E}, 132 \mathrm{~m}$ a.s.l.) is a semi-open habitat with a moist microclimate, situated near an old arm of the Danube River, with a fluctuating water level.

S3 - a shrub-meadow ecotone located in the Dunajské Luhy Protected Landscape Area $\left(48^{\circ} 06^{\prime} 21.13^{\prime \prime} \mathrm{N}, 17^{\circ} 10^{\prime} 10.57^{\prime \prime} \mathrm{E}, 131\right.$ $\mathrm{m}$ a.s.l.) is at the edge of a floodplain forest, with a medium-deep soil $(30-60 \mathrm{~cm})$.

S4 - is near the waste incinerator in Bratislava $\left(48^{\circ} 6^{\prime} 24.12^{\prime \prime} \mathrm{N}\right.$, $17^{\circ} 10^{\prime} 9.68^{\prime \prime} \mathrm{E}, 132 \mathrm{~m}$ a.s.l.) and is a semi-open xerothermic habitat on a gravel bar, with a shallow layer of soil (up to $30 \mathrm{~cm}$ ).

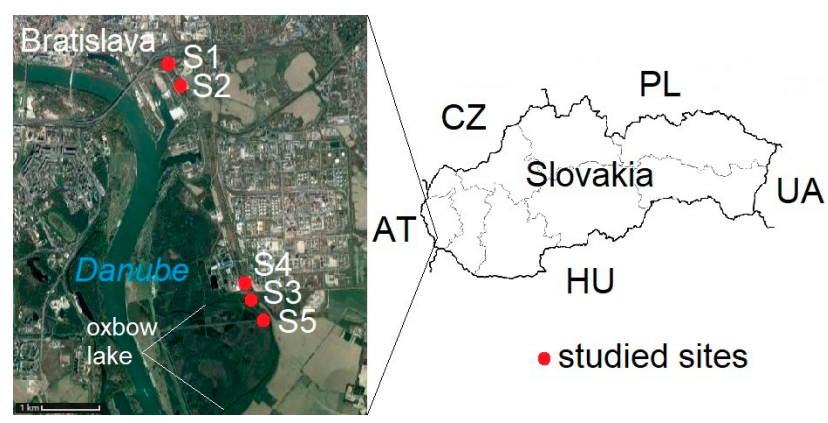

Fig. 1. Aerial photograph and map showing the areas studied in the Danube River floodplain forests in Bratislava (Slovakia): S1-S5 sites studied; AT - Austria; CZ - Czech Republic; HU - Hungary; $\mathrm{PL}$ - Poland; UA - Ukraine.

S5 - a hardwood floodplain forest, with a closed habitat and moist microclimate, located near the Biskupické Rameno oxbow lake $\left(48^{\circ} 06^{\prime} 8.88^{\prime \prime} \mathrm{N}, 17^{\circ} 10^{\prime} 29.77^{\prime \prime} \mathrm{E}, 130 \mathrm{~m}\right.$ a.s.1.).

For a better comparison, three locations in Serbia geomorphologically and botanically similar to those monitored in Slovakia (Fig. 2) were selected. The Begej River (a tributary of the Tisza) is the smallest of the rivers monitored. The mean anuual temperature in the proximity of Zrenjanin (from 1991 to 2010), which is close to these areas, was $11.9^{\circ} \mathrm{C}$, the average annual relative humidity $73.0 \%$ and mean annual total precipitation $607.0 \mathrm{~mm}$ (Republic Hydrometeorological Service of Serbia, 1999-2010).

Two sites (S6 and S7) on the alluvium of the Tisza River within the Ritovi Donjeg Potisja Special Nature Reserve, a protected area $5 \mathrm{~km}$ from the village of Aradac, close to the town of Zrenjanin, were studied.

S6 - a closed habitat with moist microclimate, close to an oxbow lake on the Tisza River $\left(45^{\circ} 22^{\prime} 50.76^{\prime \prime} \mathrm{N}, 20^{\circ} 13^{\prime} 40.83^{\prime \prime} \mathrm{E}\right.$, $74 \mathrm{~m}$ a.s.1.), which is frequently flooded in spring.

S7 - a meadow-wetland ecotone located close to the dyke on the Tisza River $\left(45^{\circ} 23^{\prime} 25.07^{\prime \prime} \mathrm{N}, 20^{\circ} 13^{\prime} 16.15^{\prime \prime} \mathrm{E}, 71 \mathrm{~m}\right.$ a.s.1.).

The third site in Serbia (S8) is located in the protected area Carska Bara Special Nature Reserve, near the village of Belo Blato, close to the town of Zrenjanin, approximately $20 \mathrm{~km}$ from the sites studied along the Tisza River.

S8 - a hardwood floodplain forest located near the Begej River $\left(45^{\circ} 16^{\prime} 41.60^{\prime \prime} \mathrm{N}, 20^{\circ} 24^{\prime} 58.56^{\prime \prime} \mathrm{E}, 73 \mathrm{~m}\right.$ a.s.1.) close to the Stari Begej oxbow lake.

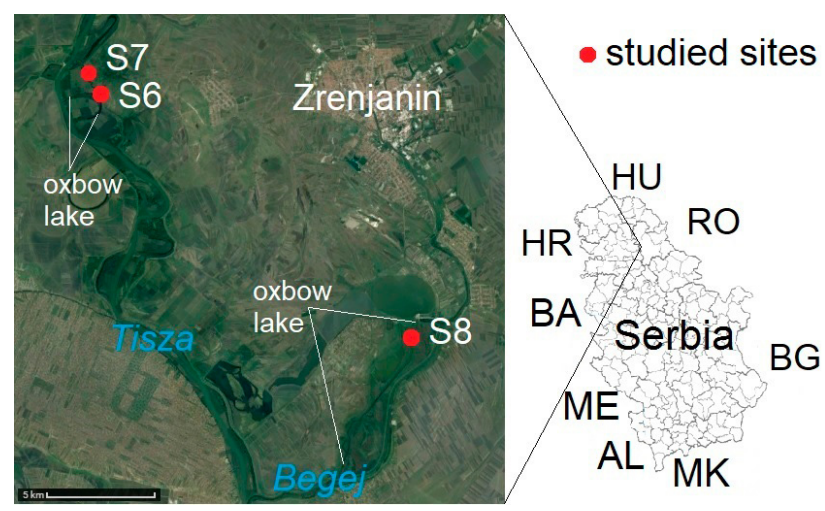

Fig. 2. Aerial photograph and map showing the areas studied in the floodplain forests near the Begej and Tisza Rivers (Serbia): S6-S8 - sites studied; AL - Albania; BA - Bosnia and Herzegovina; BG - Bulgaria; HR - Croatia; HU - Hungary; ME - Montenegro; MK Republic of North Macedonia; RO - Romania. 
More detailed information on the vegetation at these sites is presented in Litavský et al. $(2018,2021)$.

\section{Methods}

This research was carried out from February 2015 until November 2016. Ground beetles were captured using pitfall traps (Stašiov, 2015) consisting of plastic cups (diameter of opening 9 $\mathrm{cm}$ and volume 0.51 ) containing $1 \%$ formaldehyde as a fixative. At each site, five traps were set in a line at a distance of $5 \mathrm{~m}$ from each other. The traps were operational from February 2015 to November 2016 and emptied and refilled approximately at halfmonthly intervals. The contents of the five traps at each site were pooled, which formed the sample for that date. The samples were sorted in the laboratory and the ground beetles subsequently identified to species according to Trautner \& Geigenmüller (1987), Hůrka (1996) and Müller-Motzfeld (2004). Specimens were fixed in $75 \%$ ethyl alcohol and deposited in the collection of the Faculty of Natural Sciences of the Comenius University in Bratislava. A list of species was drawn up following Lorenz (2019). In Table 1 , for each species, we added its ecological characteristics. Farkač et al. (2006) classify carabids into three groups: relict (R), adaptable (A) and eurytopic (E), based on their ecological valence and association with a particular habitat. Following Šustek (2000, 2004a, 2010, 2012), the humidity preferences of ground beetles were classified as either; 1 - strongly xerophilous, $2-3$-intermediate between strongly xerophilous and mesohygrophilous, 4-5 mesohygrophilous, 6-7 - intermediate between mesohygrophilous and strongly hygrophilous, or 8 - strongly hygrophilous. In terms of dispersal ability, species were divided into three groups: (i) non-flying, (ii) flying and (iii) brachypterous, which occasionally fly (Hůrka, 1992, 1996; Matalin, 2003; Šustek, 2012).

Samples of soil and leaf litter for chemical analyses were collected on June 8th 2016. More detailed information on the methods of analysis of the soil and leaf litter samples is given in Litavský et al. $(2018,2021)$.

We defined four levels of anthropogenic effect on the study area: level 1 - with minimum disturbance; level 2 - with grazing; level 3 - with infrequent movement of vehicles and minor solid waste pollution; level 4 - with solid waste pollution, frequent mowing and cutting and building activities.

Of the environmental factors that could affect the composition of carabid communities we recorded species richness in individual layers, stand canopy of individual layers, anthropogenic effect, area of fragment, distance from another fragment (edge), length of the periphery of the fragment, age of trees at the sites and average depth of leaf litter, which are presented in Litavský et al. $(2018,2021)$.

During the vegetation period, we recorded plant species diversity in three vegetation layers $\left(\mathrm{E}_{1}, \mathrm{E}_{2}, \mathrm{E}_{3}\right)$, as well as the covers of the tree, shrub and herbaceous plant layers, in both countries and within sites of constant size $\left(400 \mathrm{~m}^{2}\right)$. More information on the method of investigation of the vegetation is given in Litavský et al. (2021).

\section{Data analysis}

We calculated the diversity of the carabid communities in terms of species richness, Shannon diversity (Spellerberg \& Fedor, 2003) and evenness (Jost, 2006; Tuomisto, 2012; Fedor \& Zvaríková, 2018). Species epigeic activity was the mean number of individuals caught per trap per day. Carabid community composition is summarized in the species-by-site matrix of epigeic activities.

Due to the relatively low number of sites sampled and the high number of environmental variables, we did not build models describing diversity and species composition, but adopted an ex- ploratory approach and investigated all species-environment associations.

In order to investigate the diversity characteristics of communities (total epigeic activity, species richness, Shannon diversity and evenness), we produced a matrix of pairwise Spearman's correlation coefficients between environmental variables and the diversity characteristics.

Non-metric multidimensional scaling (NMDS) based on BrayCurtis distance was used to visualize similarities in the composition of carabid communities. The vectors of environmental variables were projected onto ordinations in the directions of their maximum correlations with the configuration of sites. The statistical significance of fitted environmental vectors was evaluated using permutation tests $(10,000$ permutations).

This exploratory approach allowed us to investigate all speciesenvironment associations without the risk of missing any important information caused, e.g., by the removal of highly correlated variables. Indeed, the results of such a heuristic analysis should be considered carefully and regarded as a process for generating hypotheses rather than a generalization to wider populations.

The analyses were performed in R (R Core Team, 2016) using the Hmisc (Harrell, 2016) and the Vegan libraries (Oksanen et al., 2016).

\section{RESULTS AND DISCUSSION}

During this research, a total of 2,495 adult carabids belonging to 22 tribes, 48 genera and 110 species were recorded. Seventy-nine species of ground beetles are recorded at the sites in Slovakia and 58 species at those in Serbia (Majzlan \& Litavský, 2017), with 27 of the species occurring in both countries. The values of the total epigeic activity of ground beetles captured at individual sites during this research are shown in Table 1 . The most abundant species was Agonum fuliginosum (Panzer, 1809) (487 adults recorded), which was recorded most abundantly near the oxbow lake on the Danube River (Biskupické Rameno) (S5). The other dominant species were Nebria brevicollis (Fabricius, 1792) (247 adults), Abax parallelepipedus (Piller \& Mitterpacher, 1783) (185 adults), Carabus granulatus Linnaeus, 1758 (125 adults), Pterostichus niger (Schaller, 1783) (117 adults), Patrobus atrorufus (Strøm, 1768) (114 adults), Pterostichus melanarius (Illiger, 1798) (108 adults), Carabus coriaceus Linnaeus, 1758 (105 adults) and Agonum micans (Nicolai, 1822) (102 adults). No species was recorded at all the sites studied. The largest number of adult individuals was recorded at site S2 (967), which is a semi-open habitat with a moist microclimate and where the highest species richness of ground beetles was recorded (38 species). The lowest species richness of carabids was recorded at $\mathrm{S} 4$, which is a semi-open xerothermic habitat (23 species). In addition, we divided the ground beetles into different groups according to several criteria (Table 1). Based on their ecological valence and association with a particular habitat, we recorded 10 relict, 38 eurytopic and 62 adaptable species of carabids. In terms of their humidity preferences 24 were strongly xerophilous, 39 mesohygrophilous and 47 strongly hygrophilous species. With regard to their ability to fly, we recorded 20 brachypterous species that occasionally fly, 20 non-flying species and 70 species of ground beetles capable of flying. 
Table 1. A list of the species of carabids collected, the total numbers of specimens and species, diversity measures of carabid communities recorded at the sites studied and their division according to ecological characteristics: Abbr. - abbreviations; * - published in Majzlan \& Litavský (2017); EV - ecological valence of carabids and their association with habitats: R (relict), A (adaptable), E (eurytopic); HP - humidity preferences of carabids recorded: 1 - strongly xerophilous, 2-3 - intermediate between strongly xerophilous and mesohygrophilous, 4-5 - mesohygrophilous, 6-7 - intermediate between mesohygrophilous and strongly hygrophilous, 8 - strongly hygrophilous; FL - ability to fly: F (flying), N (non-flying), B (brachypterous, occasionally able to fly).

\begin{tabular}{|c|c|c|c|c|c|c|c|c|c|c|c|c|c|}
\hline \multirow{2}{*}{ Species } & \multirow{2}{*}{ Abbr. } & \multirow{2}{*}{ EV } & \multirow{2}{*}{ HP } & \multirow{2}{*}{$\mathrm{FL}$} & & & & & & & & & \\
\hline & & & & & S1 & S2 & S3 & S4 & S5 & $\mathrm{S}^{*}$ & $\mathrm{~S} 7^{*}$ & S8* & specimens \\
\hline Abax carinatus (Duftschmid, 1812) & Abca & A & 5 & $\mathrm{~N}$ & 0 & 0 & 0 & 0 & 0 & 3 & 0 & 0 & 3 \\
\hline Abax ovalis (Duftschmid, 1812) & Abov & A & 6 & $\mathrm{~N}$ & 6 & 5 & 0 & 0 & 4 & 0 & 0 & 0 & 15 \\
\hline Abax parallelepipedus (Piller \& Mitterpacher, 1783) & Abpa & $A$ & 3 & $\mathrm{~N}$ & 45 & 2 & 12 & 0 & 124 & 2 & 0 & 0 & 185 \\
\hline Acupalpus flavicollis (Sturm, 1825) & Acfl & A & 6 & $\mathrm{~F}$ & 0 & 0 & 0 & 0 & 0 & 17 & 0 & 0 & 17 \\
\hline Acupalpus meridianus (Linnaeus, 1760) & Acme & $E$ & 6 & $\mathrm{~F}$ & 0 & 0 & 1 & 0 & 1 & 0 & 0 & 0 & 2 \\
\hline Agonum duftschmidi Schmidt, 1994 & Agdu & $\mathrm{A}$ & 8 & $\mathrm{~F}$ & 0 & 4 & 0 & 0 & 4 & 0 & 0 & 0 & 8 \\
\hline Agonum fuliginosum (Panzer, 1809) & Agfu & A & 8 & $\mathrm{~F}$ & 0 & 316 & 0 & 0 & 16 & 155 & 0 & 0 & 487 \\
\hline Agonum lugens (Duftschmid, 1812) & Aglu & $\mathrm{R}$ & 8 & $\mathrm{~F}$ & 0 & 21 & 0 & 0 & 0 & 0 & 0 & 0 & 21 \\
\hline Agonum marginatum (Linnaeus, 1758) & Agma & A & 8 & $\mathrm{~F}$ & 1 & 2 & 0 & 0 & 0 & 0 & 0 & 0 & 3 \\
\hline Agonum micans (Nicolai, 1822) & Agmi & A & 7 & $\mathrm{~F}$ & 0 & 102 & 0 & 0 & 0 & 0 & 0 & 0 & 102 \\
\hline Agonum nigrum Dejean, 1828 & Agni & $\mathrm{R}$ & 5 & $\mathrm{~F}$ & 0 & 5 & 0 & 0 & 0 & 0 & 0 & 0 & 5 \\
\hline Agonum piceum (Linnaeus, 1758) & Agpi & A & 8 & $\mathrm{~F}$ & 0 & 0 & 0 & 0 & 0 & 13 & 0 & 1 & 14 \\
\hline Agonum sexpunctatum (Linnaeus, 1758) & Agse & A & 5 & $\mathrm{~F}$ & 0 & 7 & 0 & 0 & 0 & 0 & 0 & 0 & 7 \\
\hline Amara aenea (De Geer, 1774) & Amae & $E$ & 3 & $\mathrm{~F}$ & 0 & 0 & 1 & 0 & 5 & 0 & 0 & 0 & 6 \\
\hline Amara aulica (Panzer, 1796) & Amau & $\mathrm{E}$ & 3 & $\mathrm{~F}$ & 0 & 0 & 0 & 0 & 0 & 0 & 2 & 0 & 2 \\
\hline Amara eurynota (Panzer, 1796) & Ameu & $\bar{E}$ & 3 & $\mathrm{~F}$ & 0 & 0 & 0 & 0 & 0 & 0 & 4 & 0 & 4 \\
\hline Amara ovata (Fabricius, 1792) & Amov & $\mathrm{E}$ & 3 & $\mathrm{~F}$ & 0 & 0 & 1 & 0 & 0 & 0 & 1 & 0 & 2 \\
\hline Amara saphyrea Dejean, 1828 & Amsa & $\bar{A}$ & 3 & $\mathrm{~F}$ & 0 & 0 & 0 & 2 & 1 & 0 & 0 & 0 & 3 \\
\hline Anchomenus dorsalis (Pontoppidan, 1763) & Ando & $E$ & 3 & $\mathrm{~F}$ & 0 & 3 & 1 & 0 & 0 & 1 & 0 & 0 & 5 \\
\hline Anisodactylus signatus (Panzer, 1796) & Ansi & $E$ & 5 & $\mathrm{~F}$ & 0 & 0 & 0 & 1 & 1 & 0 & 0 & 0 & 2 \\
\hline Asaphidion austriacum Schweiger, 1975 & Asau & A & 6 & $\mathrm{~F}$ & 0 & 3 & 0 & 0 & 0 & 0 & 0 & 0 & 3 \\
\hline Asaphidion flavipes (Linnaeus, 1761) & Asfl & $E$ & 6 & $\mathrm{~F}$ & 0 & 2 & 0 & 0 & 0 & 0 & 0 & 0 & 2 \\
\hline Badister bullatus (Schrank, 1798) & Babu & $\mathrm{A}$ & 5 & $\mathrm{~F}$ & 0 & 0 & 0 & 0 & 0 & 1 & 0 & 0 & 1 \\
\hline Badister dorsiger (Duftschmid, 1812) & Bado & $\mathrm{R}$ & 6 & $\mathrm{~F}$ & 0 & 0 & 0 & 0 & 0 & 2 & 0 & 0 & 2 \\
\hline Badister lacertosus Sturm, 1815 & Bala & A & 6 & $\mathrm{~F}$ & 0 & 0 & 0 & 0 & 0 & 1 & 12 & 1 & 14 \\
\hline Badister sodalis (Duftschmid, 1812) & Baso & A & 7 & $\mathrm{~F}$ & 0 & 0 & 0 & 2 & 0 & 0 & 0 & 0 & 2 \\
\hline Bembidion dentellum (Thunberg, 1787) & Bede & A & 8 & $\mathrm{~F}$ & 0 & 2 & 0 & 0 & 2 & 0 & 0 & 0 & 4 \\
\hline Bembidion femoratum Sturm, 1825 & Befe & $E$ & 7 & $\mathrm{~F}$ & 3 & 0 & 0 & 0 & 1 & 0 & 0 & 0 & 4 \\
\hline Bembidion lampros (Herbst, 1784) & Bela & $\mathrm{E}$ & 3 & B & 0 & 2 & 0 & 0 & 0 & 0 & 0 & 0 & 2 \\
\hline Bembidion litorale (Olivier, 1790) & Beli & $\mathrm{R}$ & 6 & $\mathrm{~F}$ & 1 & 0 & 0 & 0 & 0 & 0 & 0 & 0 & 1 \\
\hline Bembidion semipunctatum (Donovan, 1806) & Bese & A & 8 & $\mathrm{~F}$ & 0 & 0 & 0 & 0 & 0 & 1 & 0 & 0 & 1 \\
\hline Bembidion varium (Olivier, 1795) & Beva & $E$ & 8 & $\mathrm{~F}$ & 0 & 1 & 0 & 0 & 0 & 0 & 0 & 2 & 3 \\
\hline Blemus discus (Fabricius, 1792) & Bldi & $\mathrm{A}$ & 6 & $\mathrm{~F}$ & 0 & 2 & 0 & 0 & 0 & 0 & 0 & 1 & 3 \\
\hline Bradycellus caucasicus (Chaudoir, 1846) & Brca & A & 3 & $\mathrm{~N}$ & 0 & 0 & 1 & 0 & 0 & 0 & 0 & 0 & 1 \\
\hline Bradycellus ruficollis (Stephens, 1828) & Brru & $\mathrm{R}$ & 7 & $\mathrm{~F}$ & 0 & 0 & 0 & 0 & 0 & 0 & 1 & 0 & 1 \\
\hline Brachinus crepitans (Linnaeus, 1758) & $\mathrm{Brcr}$ & $\mathrm{E}$ & 3 & $\mathrm{~F}$ & 0 & 0 & 1 & 0 & 0 & 0 & 0 & 0 & 1 \\
\hline Brachinus explodens Duftschmid, 1812 & Brex & $\bar{E}$ & 3 & $\mathrm{~F}$ & 0 & 0 & 0 & 0 & 0 & 0 & 1 & 0 & 1 \\
\hline Broscus cephalotes (Linnaeus, 1758) & Brce & A & 3 & $\mathrm{~F}$ & 0 & 0 & 0 & 1 & 0 & 0 & 0 & 0 & 1 \\
\hline Calathus erratus (Sahlberg, 1827) & Caer & A & 4 & B & 4 & 0 & 6 & 6 & 5 & 0 & 0 & 0 & 21 \\
\hline Calathus fuscipes (Goeze, 1777) & Cafu & $E$ & 4 & B & 0 & 0 & 2 & 0 & 8 & 0 & 5 & 0 & 15 \\
\hline Calathus melanocephalus (Linnaeus, 1758) & Came & $\mathrm{E}$ & 3 & $\mathrm{~B}$ & 0 & 2 & 1 & 5 & 6 & 5 & 6 & 3 & 28 \\
\hline Calosoma auropunctatum (Herbst, 1784) & Caau & $\bar{A}$ & 3 & $\mathrm{~F}$ & 0 & 0 & 0 & 0 & 1 & 0 & 0 & 0 & 1 \\
\hline Callistus Iunatus (Fabricius, 1775) & Calu & A & 3 & $\mathrm{~F}$ & 0 & 0 & 0 & 0 & 0 & 1 & 0 & 0 & 1 \\
\hline Carabus cancellatus Illiger, 1798 & Caca & A & 4 & $\mathrm{~N}$ & 0 & 0 & 3 & 4 & 18 & 31 & 2 & 1 & 59 \\
\hline Carabus clathratus Linnaeus, 1760 & Cacl & $\mathrm{R}$ & 8 & $\mathrm{~N}$ & 0 & 0 & 0 & 0 & 0 & 0 & 0 & 5 & 5 \\
\hline Carabus convexus Fabricius, 1775 & Cacon & $A$ & 4 & $\mathrm{~N}$ & 0 & 0 & 0 & 0 & 0 & 0 & 1 & 0 & 1 \\
\hline Carabus coriaceus Linnaeus, 1758 & Caco & A & 5 & $\mathrm{~N}$ & 4 & 0 & 17 & 10 & 20 & 11 & 35 & 8 & 105 \\
\hline Carabus granulatus Linnaeus, 1758 & Cagr & $E$ & 7 & $\mathrm{~B}$ & 0 & 0 & 0 & 0 & 0 & 123 & 2 & 0 & 125 \\
\hline Carabus hortensis Linnaeus, 1758 & Caho & $A$ & 4 & $\mathrm{~N}$ & 2 & 0 & 0 & 0 & 0 & 0 & 0 & 0 & 2 \\
\hline Carabus intricatus Linnaeus, 1760 & Cain & A & 4 & $\mathrm{~N}$ & 3 & 0 & 0 & 0 & 0 & 0 & 0 & 0 & 3 \\
\hline Carabus scheidleri Panzer, 1799 & Casc & A & 5 & $\mathrm{~N}$ & 0 & 0 & 0 & 0 & 1 & 0 & 0 & 0 & 1 \\
\hline Carabus ullrichii Germar, 1824 & Caul & A & 4 & $\mathrm{~N}$ & 0 & 0 & 2 & 3 & 38 & 0 & 0 & 0 & 43 \\
\hline Carabus violaceus Linnaeus, 1758 & Cavi & A & 4 & $\mathrm{~N}$ & 0 & 0 & 3 & 3 & 32 & 2 & 9 & 4 & 53 \\
\hline Chlaenius festivus (Panzer, 1796) & Chfe & A & 7 & $\mathrm{~F}$ & 0 & 0 & 0 & 0 & 0 & 0 & 0 & 1 & 1 \\
\hline Chlaenius tristis (Schaller, 1783) & Chtr & $A$ & 8 & $\mathrm{~F}$ & 0 & 0 & 0 & 0 & 0 & 0 & 4 & 0 & 4 \\
\hline Cicindela germanica Linnaeus, 1758 & Cige & A & 3 & $\mathrm{~F}$ & 0 & 0 & 0 & 0 & 0 & 0 & 2 & 0 & 2 \\
\hline Clivina collaris (Herbst, 1784) & Clco & $E$ & 6 & $\mathrm{~F}$ & 0 & 1 & 0 & 0 & 0 & 0 & 0 & 0 & $\overline{1}$ \\
\hline Clivina fossor (Linnaeus, 1758) & Clfo & $\mathrm{E}$ & 6 & $\mathrm{~B}$ & 0 & 0 & 0 & 0 & 0 & 12 & 0 & 0 & 12 \\
\hline Demetrias atricapillus (Linnaeus, 1758) & Deat & $E$ & 4 & $\mathrm{~F}$ & 1 & 0 & 0 & 0 & 0 & 0 & 0 & 0 & 1 \\
\hline Diachromus germanus (Linnaeus, 1758) & Dige & A & 7 & $\mathrm{~F}$ & 0 & 0 & 0 & 0 & 2 & 0 & 0 & 1 & 3 \\
\hline Dromius agilis (Fabricius, 1787) & Drag & A & 5 & $\mathrm{~F}$ & 0 & 0 & 0 & 1 & 0 & 0 & 0 & 0 & 1 \\
\hline Dromius quadrimaculatus (Linnaeus, 1758) & Drqu & A & 4 & $\mathrm{~F}$ & 0 & 0 & 0 & 0 & 0 & 0 & 0 & 1 & 1 \\
\hline Drypta dentata (Rossi, 1790) & Drde & $\mathrm{E}$ & 4 & $\mathrm{~F}$ & 0 & 0 & 0 & 1 & 0 & 0 & 0 & 0 & 1 \\
\hline Dyschirius globosus (Herbst, 1784) & Dygl & $\mathrm{E}$ & 8 & $\mathrm{~B}$ & 0 & 1 & 0 & 0 & 0 & 33 & 0 & 0 & 34 \\
\hline Elaphrus riparius (Linnaeus, 1758) & Elri & $\mathrm{E}$ & 8 & $\mathrm{~F}$ & 0 & 2 & 0 & 0 & 0 & 0 & 0 & 0 & 2 \\
\hline Elaphrus uliginosus Fabricius, 1792 & Elul & $\mathrm{A}$ & 8 & $\mathrm{~F}$ & 0 & 0 & 0 & 0 & 0 & 1 & 0 & 2 & 3 \\
\hline Epaphius secalis (Paykull, 1790) & Epse & A & 6 & $\mathrm{~N}$ & 0 & 0 & 3 & 2 & 0 & 0 & 0 & 0 & 5 \\
\hline Epaphius rivularis (Gyllenhal, 1810) & Epri & $\mathrm{R}$ & 8 & $\mathrm{~N}$ & 0 & 0 & 0 & 0 & 0 & 0 & 0 & 3 & 3 \\
\hline Harpalus affinis (Schrank, 1781) & Haaf & $E$ & 3 & $\mathrm{~F}$ & 0 & 0 & 0 & 0 & 0 & 0 & 1 & 0 & 1 \\
\hline Harpalus albanicus Reitter, 1900 & Haal & $\mathrm{R}$ & 2 & $\mathrm{~F}$ & 0 & 0 & 2 & 1 & 0 & 0 & 0 & 0 & 3 \\
\hline Harpalus distinguendus (Duftschmid, 1812) & Hadi & $E$ & 3 & $\mathrm{~F}$ & 0 & 0 & 0 & 0 & 0 & 2 & 0 & 0 & 2 \\
\hline Harpalus griseus (Panzer, 1796) & Hagr & $E$ & 5 & $\mathrm{~F}$ & 0 & 0 & 0 & 0 & 0 & 0 & 0 & 1 & 1 \\
\hline
\end{tabular}


Table 1 (continued).

\begin{tabular}{|c|c|c|c|c|c|c|c|c|c|c|c|c|c|}
\hline \multirow{2}{*}{ Species } & \multirow{2}{*}{ Abbr. } & \multirow{2}{*}{ EV } & \multirow{2}{*}{ HP } & \multirow{2}{*}{ FL } & \multicolumn{8}{|c|}{ Sites } & \multirow{2}{*}{$\begin{array}{c} \\
\text { specimens } \\
\end{array}$} \\
\hline & & & & & S1 & S2 & S3 & S4 & S5 & S6* & $S 7^{*}$ & S8* & \\
\hline Harpalus latus (Linnaeus, 1758) & Hala & A & 4 & $\mathrm{~F}$ & 1 & 1 & 6 & 4 & 8 & 0 & 0 & 0 & 20 \\
\hline Harpalus rufipes (De Geer, 1774) & Haru & $E$ & 4 & $\mathrm{~F}$ & 1 & 0 & 2 & 3 & 37 & 3 & 5 & 0 & 51 \\
\hline Laemostenus punctatus (Dejean, 1828) & Latepu & A & 4 & $\mathrm{~N}$ & 0 & 0 & 0 & 0 & 12 & 0 & 3 & 0 & 15 \\
\hline Leistus ferrugineus (Linnaeus, 1758) & Lefe & $E$ & 4 & $\mathrm{~F}$ & 0 & 0 & 1 & 0 & 4 & 0 & 3 & 0 & 8 \\
\hline Leistus piceus Frölich, 1799 & Lepi & $\bar{A}$ & 6 & B & 0 & 0 & 0 & 0 & 0 & 1 & 1 & 3 & 5 \\
\hline Leistus rufomarginatus (Duftschmid, 1812) & Leru & $\mathrm{R}$ & 5 & $\mathrm{~F}$ & 0 & 0 & 0 & 0 & 1 & 0 & 0 & 0 & 1 \\
\hline Loricera pilicornis (Fabricius, 1775) & Lopi & $E$ & 4 & $\mathrm{~F}$ & 1 & 2 & 0 & 0 & 0 & 0 & 0 & 5 & 8 \\
\hline Microlestes maurus (Sturm, 1827) & Mima & $\mathrm{E}$ & 2 & $\mathrm{~B}$ & 0 & 0 & 0 & 0 & 2 & 0 & 0 & 0 & 2 \\
\hline Molops piceus (Panzer, 1793) & Mopi & $\bar{A}$ & 4 & $\mathrm{~N}$ & 0 & 1 & 0 & 0 & 0 & 0 & 0 & 1 & 2 \\
\hline Nebria brevicollis (Fabricius, 1792) & Nebr & $A$ & 6 & $\mathrm{~F}$ & 3 & 218 & 0 & 0 & 15 & 5 & 0 & 6 & 247 \\
\hline Notiophilus biguttatus (Fabricius, 1779) & Nobi & $A$ & 4 & $\mathrm{~B}$ & 1 & 3 & 2 & 0 & 3 & 0 & 0 & 0 & 9 \\
\hline Notiophilus palustris (Duftschmid, 1812) & Nopa & $E$ & 4 & $B$ & 0 & 0 & 0 & 0 & 0 & 2 & 0 & 0 & 2 \\
\hline Oodes helopioides (Fabricius, 1792) & Oohe & A & 8 & $\mathrm{~F}$ & 0 & 2 & 0 & 0 & 0 & 1 & 0 & 0 & 3 \\
\hline Ophonus azureus (Fabricius, 1775) & Opaz & $\mathrm{E}$ & 2 & $\mathrm{~F}$ & 0 & 0 & 0 & 0 & 0 & 1 & 37 & 0 & 38 \\
\hline Ophonus puncticollis (Paykull, 1798) & Oppu & $\bar{A}$ & 2 & $\mathrm{~F}$ & 0 & 0 & 0 & 2 & 0 & 0 & 0 & 0 & 2 \\
\hline Oxypselaphus obscurus (Herbst, 1784) & Oxob & A & 7 & $B$ & 4 & 1 & 4 & 2 & 5 & 42 & 0 & 0 & 58 \\
\hline Paradromius linearis (Olivier, 1795) & Pali & $\mathrm{E}$ & 2 & $\mathrm{~B}$ & 1 & 0 & 1 & 0 & 1 & 0 & 0 & 0 & 3 \\
\hline Patrobus atrorufus (Strøm, 1768) & Paat & A & 7 & $\mathrm{~B}$ & 0 & 85 & 3 & 0 & 0 & 20 & 6 & 0 & 114 \\
\hline Platyderus rufus (Duftschmid, 1812) & Plru & $A$ & 3 & $\mathrm{~N}$ & 1 & 37 & 0 & 3 & 2 & 8 & 0 & 0 & 51 \\
\hline Platynus assimilis (Paykull, 1790) & Plas & $A$ & 7 & $\mathrm{~F}$ & 2 & 14 & 0 & 0 & 0 & 46 & 0 & 0 & 62 \\
\hline Platynus livens (Gyllenhal, 1810) & Plli & $\mathrm{R}$ & 8 & $\mathrm{~F}$ & 0 & 1 & 0 & 0 & 0 & 0 & 0 & 0 & 1 \\
\hline Poecilus cupreus (Linnaeus, 1758) & Pocu & $\mathrm{E}$ & 4 & $\mathrm{~F}$ & 0 & 6 & 0 & 12 & 0 & 0 & 0 & 0 & 18 \\
\hline Pterostichus anthracinus (Illiger, 1798) & Ptan & A & 8 & $\mathrm{~B}$ & 0 & 2 & 0 & 0 & 0 & 0 & 0 & 0 & 2 \\
\hline Pterostichus macer (Marsham, 1802) & Ptma & $A$ & 4 & $\mathrm{~F}$ & 0 & 0 & 0 & 0 & 0 & 0 & 0 & 1 & 1 \\
\hline Pterostichus melanarius (Illiger, 1798) & Ptme & $\mathrm{E}$ & 5 & $\mathrm{~B}$ & 11 & 59 & 5 & 0 & 4 & 28 & 1 & 0 & 108 \\
\hline Pterostichus niger (Schaller, 1783) & Ptni & $A$ & 6 & $\mathrm{~F}$ & 14 & 42 & 0 & 0 & 30 & 30 & 0 & 1 & 117 \\
\hline Pterostichus nigrita (Paykull, 1790) & Ptnigr & $\mathrm{E}$ & 8 & $\mathrm{~B}$ & 5 & 0 & 0 & 0 & 21 & 0 & 0 & 0 & 26 \\
\hline Pterostichus oblongopunctatus (Fabricius, 1787) & Ptob & A & 5 & $\mathrm{~N}$ & 0 & 3 & 0 & 0 & 0 & 0 & 0 & 0 & 3 \\
\hline Pterostichus strenuus (Panzer, 1796) & Ptst & $\mathrm{E}$ & 7 & $\mathrm{~B}$ & 0 & 0 & 2 & 0 & 0 & 0 & 0 & 0 & 2 \\
\hline Pterostichus vernalis (Panzer, 1796) & Ptve & $\mathrm{A}$ & 8 & $\mathrm{~F}$ & 0 & 0 & 1 & 1 & 0 & 0 & 0 & 0 & 2 \\
\hline Stenolophus mixtus (Herbst, 1784) & Stmi & A & 8 & $\mathrm{~F}$ & 0 & 0 & 0 & 0 & 0 & 2 & 0 & 1 & 3 \\
\hline Stenolophus skrimshiranus Stephens, 1828 & Stsk & A & 4 & $\mathrm{~F}$ & 0 & 0 & 0 & 0 & 0 & 0 & 0 & 1 & 1 \\
\hline Syntomus obscuroguttatus (Duftschmid, 1812) & Syob & A & 5 & $\mathrm{~F}$ & 0 & 1 & 0 & 1 & 0 & 0 & 0 & 0 & 2 \\
\hline Syntomus pallipes Dejean, 1825 & Sypa & A & 5 & B & 0 & 0 & 0 & 2 & 0 & 0 & 0 & 0 & 2 \\
\hline Tachys bistriatus (Duftschmid, 1812) & Tabi & A & 8 & $\mathrm{~F}$ & 0 & 0 & 0 & 0 & 1 & 0 & 0 & 0 & 1 \\
\hline Trechus austriacus Dejean, 1831 & Trau & $\mathrm{E}$ & 5 & $\mathrm{~B}$ & 0 & 0 & 0 & 0 & 1 & 0 & 0 & 0 & 1 \\
\hline Trechus pulchellus Putzeys, 1845 & Trpu & $A$ & 5 & $\mathrm{~N}$ & 1 & 0 & 0 & 0 & 0 & 0 & 0 & 0 & 1 \\
\hline Trechus quadristriatus (Schrank, 1781) & Trqu & $E$ & 4 & $\mathrm{~F}$ & 0 & 4 & 0 & 0 & 0 & 3 & 5 & 7 & 19 \\
\hline$\Sigma$ specimens & & & & & 116 & 967 & 84 & 72 & 437 & 609 & 149 & 61 & 2,495 \\
\hline$\Sigma$ species & & & & & 23 & 38 & 26 & 23 & 36 & 34 & 24 & 24 & 110 \\
\hline Shannon diversity index & & & & & 2.316 & 2.165 & 2.829 & 2.829 & 2.711 & 2.516 & 2.477 & 2.876 & \\
\hline Evenness & & & & & 0.739 & 0.595 & 0.868 & 0.902 & 0.756 & 0.714 & 0.779 & 0.905 & \\
\hline
\end{tabular}

The associations between diversity characteristics and environmental variables were evaluated using correlation analysis. The total epigeic activity of carabids was significantly and positively associated with the number of species of plants in the $E_{3}$ vegetation layer and the relative content of $\mathrm{N}$ in leaf litter, and negatively with the cover of the $\mathrm{E}_{1}$ layer (Table 2). Species richness was significantly positively associated with the number of species of plants in the $\mathrm{E}_{3}$ layer and the $\mathrm{pH}$ of the leaf litter, while the trend in evenness was exactly the opposite.

NMDS ordination revealed that the composition of carabid communities was significantly associated with the number of species of plants in the $\mathrm{E}_{3}$ layer and $\mathrm{pH}$ of the leaf litter (Fig. 3). Among the most active carabid species, Trechus quadristriatus (Schrank, 1781), Ophonus azureus (Fabricius, 1775), Calathus melanocephalus (Linnaeus, 1758) and C. coriaceus were typical of stands with low leaf litter pHs. Agonum micans, A. fuliginosum, Platynus assimilis (Paykull, 1790), Acupalpus flavicollis (Sturm, 1825), Dyschirius globosus (Herbst, 1784) P. atrorufus, Platyderus rufus (Duftschmid, 1812) and N. brevicollis preferred fragments with high number of species of plants in the $\mathrm{E}_{3}$ vegetation layer.
The results of our two-year research indicate that the floodplain forests and their ecotones alongside the Danube, the Tisza and the Begej Rivers provided appropriate conditions for ground beetle communities, based on their high species richness. Šustek (1994b) states that about 25-35 species of carabids are usually present at any one time at one locality under natural conditions, but in the best conserved floodplain forests the number of carabid species exceeds 40 . We recorded the highest number of carabid species at S2 (38 species), S5 (36 species) and S6 (34 species) (typical floodplain forests near oxbow lakes), indicating that they are high quality habitats for carabids. In total, we recorded 110 species of ground beetles during this study. For comparison, Šustek (2004b) records 60 species of carabids using pitfall trapping in the Jurský Šúr Nature Reserve close to Bratislava. A survey of beetles in the Litovelské Pomoraví Protected Landscape Area, within the floodplain forests on the Morava River, Nakládal (2008) revealed 93 species of ground beetles at six sites in 2006. Nakládal's (2008) objective was to record as many species as possible and therefore he used a variety collecting methods, such as beating trees and shrubs, individual sampling, sweeping, pitfall trapping, examina- 
Table 2. Matrix of Spearman correlation coefficients between sum of the diversity characteristics of carabid communities and environmental variables recorded at the sites studied in Slovakia and Serbia: s - soil; I - leaf litter; $E_{1}, E_{2}, E_{3}[\%]$ - canopy of individual vegetation layers; $\Sigma$ species $\left(E_{1}, E_{2}, E_{3}\right)$ - species richness of individual vegetation layers; $\mathrm{pH}_{\mathrm{H} 2 \mathrm{O}}$ - acidity of the supernatant of a suspension of soil and $\mathrm{H}_{2} \mathrm{O}$ (ratio $1: 2.5$ ); $\mathrm{k}$ [mS. $\mathrm{cm}^{-1}$ ] - conductivity of $\mathrm{H}_{2} \mathrm{O}$ extract ( $\mathrm{P}$ - phosphorus; $\mathrm{N}$ - nitrogen; $\mathrm{C}$ - carbon; $\mathrm{H}$ - hydrogen. Statistically significant correlations $(\alpha=5 \%)$ are highlighted in bold.

\begin{tabular}{|c|c|c|c|c|}
\hline $\begin{array}{l}\text { Environmental } \\
\text { variable }\end{array}$ & $\begin{array}{l}\text { Epigeic } \\
\text { activity }\end{array}$ & $\begin{array}{l}\text { Species } \\
\text { richness }\end{array}$ & $\begin{array}{c}\text { Shannon } \\
\text { diversity } \\
\text { index }\end{array}$ & Evenness \\
\hline Anthropological effect & 0.01 & -0.19 & -0.59 & -0.26 \\
\hline $\mathrm{E}_{1}[\%]$ & -0.75 & -0.55 & 0.12 & 0.51 \\
\hline $\mathrm{E}_{2}[\%]$ & 0.61 & 0.69 & -0.25 & -0.51 \\
\hline $\mathrm{E}_{3}[\%]$ & 0.25 & 0.07 & -0.05 & -0.22 \\
\hline$\Sigma$ species $\left(E_{1}\right)$ & -0.49 & -0.12 & 0.23 & 0.42 \\
\hline$\Sigma$ species $\left(E_{2}\right)$ & -0.54 & -0.32 & 0.02 & 0.02 \\
\hline$\Sigma$ species $\left(E_{3}\right)$ & 0.84 & 0.82 & -0.33 & -0.71 \\
\hline Area $\left[\mathrm{m}^{2}\right]$ & 0.14 & 0.06 & 0.19 & 0.05 \\
\hline Edge of area [m] & -0.17 & -0.22 & 0.38 & 0.19 \\
\hline Circuit of area [m] & 0.12 & -0.17 & 0.12 & 0.17 \\
\hline Age [year] & 0.66 & 0.40 & -0.56 & -0.66 \\
\hline Leaf litter depth [cm] & 0.60 & 0.42 & -0.16 & -0.49 \\
\hline $\mathrm{pH}_{\mathrm{H} 2 \mathrm{O}} / \mathrm{s}$ & -0.27 & 0.16 & -0.10 & -0.05 \\
\hline $\mathrm{pH}_{\mathrm{H} 2 \mathrm{O}} / \mathrm{l}$ & 0.56 & 0.75 & -0.44 & -0.73 \\
\hline $\mathrm{k}\left[\mathrm{mS} \cdot \mathrm{cm}^{-1}\right] / \mathrm{s}$ & 0.48 & 0.28 & -0.26 & -0.21 \\
\hline $\mathrm{k}\left[\mathrm{mS} . \mathrm{cm}^{-1}\right] / \mathrm{l}$ & 0.05 & -0.27 & -0.17 & 0.26 \\
\hline$P\left[\mathrm{mg} \cdot \mathrm{kg}^{-1}\right] / \mathrm{s}$ & 0.07 & -0.14 & -0.10 & 0.14 \\
\hline $\mathrm{P}\left[\mathrm{mg} \cdot \mathrm{kg}^{-1}\right] / /$ & -0.06 & -0.48 & 0.00 & 0.38 \\
\hline $\mathrm{N}[\%] / \mathrm{s}$ & 0.54 & 0.19 & -0.06 & -0.30 \\
\hline N [\%]/l & 0.71 & 0.60 & -0.38 & -0.52 \\
\hline $\mathrm{C}[\%] / \mathrm{s}$ & 0.60 & 0.53 & -0.45 & -0.69 \\
\hline C [\%]/I & -0.21 & -0.58 & -0.10 & 0.14 \\
\hline $\mathrm{H}[\%] / \mathrm{s}$ & 0.62 & 0.16 & -0.19 & -0.24 \\
\hline $\mathrm{H}[\%] / /$ & 0.00 & -0.31 & 0.14 & 0.14 \\
\hline
\end{tabular}

tion of excrement and carrion, night sampling from trees and sifting organic substrates. During a two-year research of ground beetle communities in three types of habitats in floodplain forests along the Vistula River, located near the city of Bydgoszcz, Lik (2010) recorded 79 species of carabids using pitfall trapping at 18 sites. During three-years of research on the carabid fauna in a spacious pasture on the left bank of the Tisza River, near the village of Kumane (northern Serbia), Tallósi \& Sekulić (1989) recorded 55 species of Carabidae using pitfall trapping. At approximately $25 \mathrm{~km}$ from that site, Majzlan \& Litavský (2017) recorded 46 species of ground beetles in floodplain forests along the Tisza River (our sites S6 and S7) and 24 species of carabids in floodplain forests along the Begej River (site S8) in Serbia. About $70 \mathrm{~km}$ from our study areas in Serbia, Ćurčić \& Stanković (2011) recorded 72 species of Carabidae in floodplain forests along the Sava and the Drina Rivers within the Zasavica Special Nature Reserve (northwestern Serbia). Ćurčić \& Stojanović (2011) studied the carabids in the Fruška Gora National Park (northern Serbia) near the River Danube (approximately $45 \mathrm{~km}$ from our S6-S8 sites). They recorded 64 species of Carabidae at 26 locations. Although we used only pitfall traps to collect carabids at only eight sites, it is clear from the above that we recorded a higher total species richness of carabid beetles in our study.

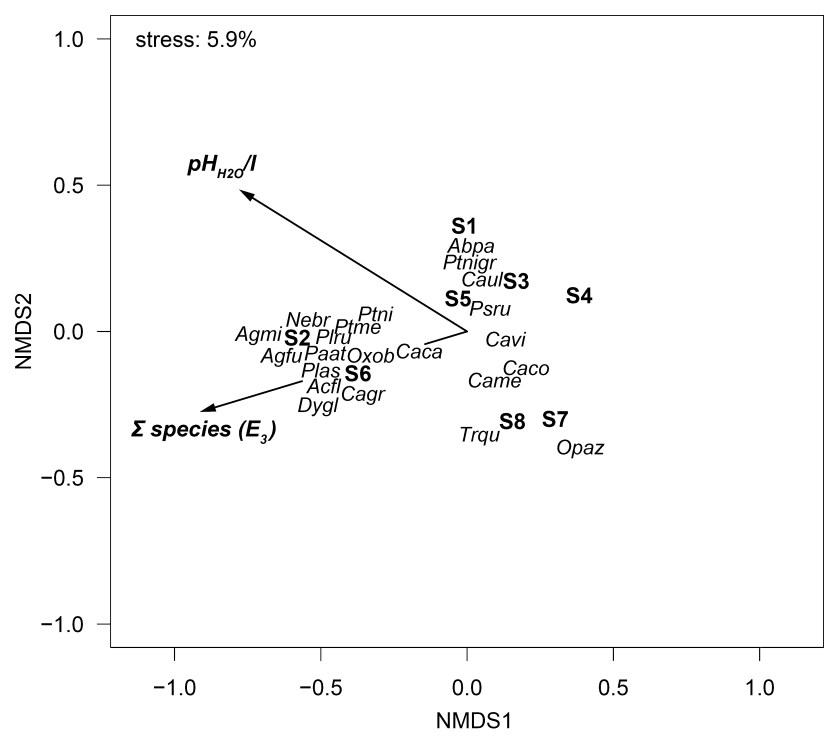

Fig. 3. Two-dimensional component of a non-metric multidimensional scaling (NMDS) of carabid communities recorded at the sites studied in Slovakia and Serbia, based on Bray-Curtis distances of epigeic activities. The vectors of significant environmental variables are fitted onto the ordination in the direction of their maximum correlation with site scores. The scores of the most active species were added as the weighted averages of site scores. Final stress value is displayed. S1-S8 - sites studied; $\Sigma$ species $\left(E_{3}\right)$ - number of species of plants in $\mathrm{E}_{3}$ vegetation layer; $\mathrm{pH}_{420} / \mathrm{l}$ - acidityof the supernatant of a suspension of leaf litter and $\mathrm{H}_{2} \mathrm{O}$ (ratio $\left.1: 2.5\right)$. For abbreviations of species names see Table 1 .

There was a statistically significant negative association between total epigeic activity of ground beetles and the cover of the herbaceous plant layer $\left(\mathrm{E}_{1}\right)$. This can be explained by the fact that density of vegetation, especially that of the herbaceous plant layer, can affect predator activity (the majority of carabids), by making it difficult for them to move on the surface of the soil. The decrease in epigeic activity of soil-dwelling beetles with increase in the density of vegetation is also reported by Heydemann (1957), Honek (1988), Humphrey et al. (1999) and Thomas et al. (2006). For example, Honek (1988) reports that some staphylinids and most species of carabids prefer sparse rather than dense stands. Zou et al. (2013), however, report that the density of herbaceous plants had little effect on the beetle activity in the Changbai Mountains.

The significant positive association between both total epigeic activity and species richness of ground beetles, and plant species richness in the $\mathrm{E}_{3}$ vegetation layer is probably due to the longer period of shading (earlier budding of different species of trees and late leaf fall), which ensure more stable and humid microclimatic conditions for a longer period, as well as a greater food supply as a high tree diversity results in a richer leaf litter. Pearce et al. (2003) also point out that some ground beetles may also benefit from increased tree species richness. Vehviläinen et al. (2008) confirm that carabids differ significantly in their preferences for stands composed of particular, yet different, species of trees. During a study of the effects of river and floodplain restoration on riparian ground beetles, Januschke \& Verdonschot (2016) revealed that the 
encroachment of woody vegetation results in a decrease in species richness of carabid beetles. According to Šustek (2005), carabid communities in forest ecosystems consist of heliophobic species that prefer areas shaded by trees or at least by dense shrub vegetation. It is cooler in the shade, which slows down the drying out of the surface of the soils and leaf litter, reduces evaporation and improves the water balance in the stand. For this reason, the association of individual species with the presence of tree cover are to some extent positively associated directly with the species' moisture requirements.

In addition to the effect of plants, ground beetle communities are also affected by properties of the soil and leaf litter. Nitrogen is an essential element for organisms. This is supported by the statistically significant positive associations between the total epigeic activity of carabids and the nitrogen content of leaf litter. The nitrogen content of the leaf litter may indirectly affect (through saprophagous prey of ground beetles) the food supply of carabid beetles. Leaf litter rich in $\mathrm{N}$ is an attractive food for saprophages that use the nitrogen in their own physiological processes (Vician et al., 2018). Therefore, this material decomposes more rapidly due to the activity of saprophages (Wittich, 1942, 1943). Dunger (1958) stresses the importance of nitrogen as the main element determining animal production and sources of food for invertebrates, including ground beetles. Vician et al. (2018) also report statistically significant associations between the content of $\mathrm{N}$ in the leaf litter and species richness, Shannon diversity and species composition of carabid beetle communities at nine stands in the Borová Hora Arboretum (Central Slovakia).

The composition of carabid beetle communities was also associated with the $\mathrm{pH}$ of the leaf litter, with a significant positive association between species richness of ground beetles and leaf litter $\mathrm{pH}$, but the opposie trend in species evenness (Table 2). Vician et al. (2018) note that a floodplain forest in which leaf litter had a high $\mathrm{pH}$ also had a high species richness of ground beetles. Magura et al. (2003) also point out that leaf litter has a positive effect on carabid species richness.

Most studies on marshes and floodplain forests record mainly hygrophilous and mesohygrophilous species of ground beetles (Šustek, 1994a, c, 2004b; Šejnohová, 2006; Lik, 2010; Igondová \& Majzlan, 2015). In the current study, there was also a predominance of hygrophilous and mesohygrophilous over xerophilous species of ground beetles, with hygrophilous species making up $42.7 \%$, mesohygrophilous $35.5 \%$ and xerophilous $21.8 \%$, i.e., a ratio of $2: 1.6: 1$, respectively.

In terms of the carabids' ecological valence and their association with a particular habitat, most (62) (56.4\%) were adaptable, 38 (34.5\%) were eurytopic and $10(9.1 \%)$ were relict species. Relict species of carabid were mostly recorded near the oxbow lakes on the Danube (S5), the Tisza (S6) and the Begej Rivers (S8). This indicates these forest stands are ecologically stable. In comparison, Igondová \& Majzlan (2015) did not record any relict species of ground beetles in the carabid communities during a one-year study of the Šuja peat bog (northern Slovakia). In relation to their ability to fly, of the species of carabid recorded (70) $(63.6 \%)$ were able to fly, $20(18.2 \%)$ were brachypterous and occasionally fly, and 20 (18.2\%) were non-flying. Arndt \& Hielscher (2007) conclude that most species of forest ground beetles are unable to fly or do not regularly fly. Šustek (2012) state that species of carabids that inhabit unstable riparian habitats are able to fly and successfully colonize anthropogenic ecosystems, such as arable land or vegetation in human settlements. Nevertheless, up to $70 \%$ of the species we recorded were able to fly and were present in closed forest stands that were mostly little or unaffected by human activity.

We determined how ground beetle communities vary in the different habitats in floodplain forests in Serbia and Slovakia and found that the total epigeic activity of carabids was significantly positively associated with the number of species of plants in the tree layer and the relative content of $\mathrm{N}$ in the leaf litter, and negatively with the cover of the herbaceous plant layer. Species richness was significantly positively associated with the number of species of plants in the tree layer and $\mathrm{pH}$ of the leaf litter, while evenness showed the opposite trend. Based on these results (Fig. 3), we selected several species of carabids, which can serve as bioindicators. We conclude that $A$. micans, $A$. fuliginosum, $P$. assimilis, A. flavicollis, D. globosus, P. atrorufus, $P$. rufus and $N$. brevicollis, which prefer forest stands with a high number of species of plants in the tree layer, can be used as bioindicators of the presence of high tree species richness in floodplain forests. We also found that T. quadristriatus, O. azureus, C. melanocephalus and C. coriaceus preferred stands in which the $\mathrm{pH}$ of the leaf litter is low and could be used as bioindicators for assessing changes in landscape structure caused by human activity resulting in soil acidification. Therefore, more information on these associations might be helpful in further elucidating how carabids respond to vegetation, soil and microclimatic conditions, and how these conditions vary in the various types of floodplain forests.

ACKNOWLEDGEMENTS. The work was supported by the research grants $1 / 0658 / 19$ and 1/0286/20 of the Slovak Grant Agency (VEGA) and by project No. 020UK-4/2020 of the Cultural and Educational Grant Agency (KEGA). The authors are grateful to the Ministry of Environmental Protection of the Republic of Serbia for permission (decision number 353-01-1529/2015-17) to collect beetles (Coleoptera) within the Carska Bara and the Ritovi Donjeg Potisja Special Nature Reserves.

\section{REFERENCES}

Ábrahámová A., Kollár J. \& ŽarnoviČAn H. 2014: Vegetation of the Váh River Inundation in the Part Nové Mesto nad Váhom - Šal'a. Comenius University, Bratislava, 50 pp. [in Slovak, English abstract].

ÁDÁM L. 1996: A check-list of the Hungarian caraboid beetles (Coleoptera). - Folia Entomol. Hung. 57: 5-64.

Antvogel H. \& Bonn A. 2001: Environmental parameters and microspatial distribution of insects: a case study of carabids in an alluvial forest. - Ecography 24: 470-482. 
ARndt E. \& Hielscher S. 2007: Ground beetles (Coleoptera: Carabidae) in the forest canopy: species composition, seasonality, and year-to-year fluctuation. In Unterseher M., Morawetz W., Klotz S. \& Arndt E. (eds): The Canopy of a Temperate Floodplain Forest: Results from Five Years of Research at the Leipzig Canopy Crane. University of Leipzig, Leipzig, pp. 106-110.

Babin-Fenske J. \& ANAND M. 2010: Terrestrial insect communities and the restoration of an industrially perturbed landscape: assessing success and surrogacy. - Restor. Ecol. 18: 73-84.

BASEDOw T.H. 1990: Effects of insecticides on Carabidae and the significance of these effects for agriculture and species number. In Stork N.E. (ed.): The Role of Ground Beetles in Ecological and Environmental Studies. Intercept, Andover, pp. 115-125.

Bishop D.J., MaJKa C.G., Bondrup-Nielsen S. \& PeCK S.B. 2009 Deadwood and saproxylic beetle diversity in naturally disturbed and managed spruce forests in Nova Scotia. - ZooKeys 22: 309-340.

ВонÁČ J. 2005: Beetles - ground beetles. In Kučera T. (ed.): Red Book on Habitats of the Czech Republic. Institute of Landscape Ecology AS CR, České Budějovice, 8 pp. URL: http:// users.prf.jcu.cz/kucert00/CKB/texty/tax_skupiny/strevlikoviti_bohac.pdf [in Czech].

BonN A. \& SCHRÖDER B. 2001: Habitat models and their transfer for single and multi species groups: a case study of carabids in an alluvial forest. - Ecography 24: 483-496.

Bouchard P., Smith A.B.T., Douglas H.B., Gimmel M.L., Brunke A.J. \& KandA K. 2017: Biodiversity of Coleoptera. In Foottit R.G. \& Adler P.H. (eds): Insect Biodiversity: Science and Society. 2nd ed. Wiley, Chichester, pp. 337-417.

ĆurČIĆ S. \& STANKović M. 2011: The ground beetles (Coleoptera: Carabidae) of the Zasavica Special Nature Reserve (Serbia). Acta Entomol. Serbica 16: 61-79.

ĆurČıć S. \& StoJanović D. 2011: New data on the carabid beetles (Coleoptera: Carabidae) of Mt. Fruška Gora (northern Serbia). - Acta Entomol. Serbica 16: 45-59.

ĆurČIć S.B., Brajković M.M. \& ĆurČIć B.P.M. 2007: The Carabids of Serbia. Institute of Zoology, Faculty of Biology, University of Belgrade, Belgrade, $1083 \mathrm{pp}$.

Ćurčić S., Pavićević D., Vesović N., Marković Đ., Petković M., Bosco F., Kuraica M. \& Nešić D. 2018: First report of aphaenopsoid trechines (Coleoptera: Carabidae: Trechini) from Serbia, with descriptions of new taxa. - Zootaxa 4425: 311-326.

De Vaate A.B., Klink A.G., Greidanus-Klaas M., Jans L.H., OOSREBAAN J. \& KOK F. 2007: Effect of habitat restoration on the macroinvertebrate fauna in a foreland along the river Waal, the main distributary in the Rhine delta. - River Res. Appl. 23: $171-183$.

Dubovský M., Fedor P., Kucharczyk H., Masarovič R. \& BALKovIČ J. 2010: Assemblages of bark-dwelling thrips (Thysanoptera) of uneven-aged oak forests in Slovakia. - Sylwan 154: 659-668.

Dunger W. 1958: Über die der Zersetzung Laubstreu durch die Boden-Makrofauna im Auenwald. — Zool. Jb. Syst. 86: 139180.

ERwIN T.L. 1991: Natural history of the carabid beetles at the BIOLAT Biological Station, Rio Manu, Pakitza, Peru. - Rev. Peru. Entomol. 33: 1-85.

Eyre M.D. \& LufF M.L. 1990: A preliminary classification of European grassland habitats using carabid beetles. In Stork N.E. (ed.): The Role of Ground Beetles in Ecological and Environmental Studies. Intercept, Andover, pp. 227-236.

FARKaČ J., KopeckÝ T. \& VeselÝ P. 2006: Carabid beetles utilization (Coleoptera: Carabidae) of Slovak fauna for quality environment indication. - Ochrana Prírody (Banská Bystrica) 25: 226-242 [in Czech, English abstract].
Fedor P. \& Zvaríková M. 2018: Conservation ecology: biodiversity indices. In Fath B.D. (ed.): Encyclopedia of Ecology. Elsevier, Amsterdam, pp. 337-346.

García M., Ortego F., Castañera P. \& Farinós G.P. 2010: Effects of exposure to the toxin Cryl Ab through Bt maize fed-prey on the performance and digestive physiology of the predatory rove beetle Atheta coriaria. - Biol. Contr. 55: 225-233.

GuÉorguiev B. 2008: New data on the ground beetles (Coleoptera: Carabidae) of Serbia. - Hist. Nat. Bulg. 19: 73-92.

GÜNTHER J. \& ASSMANN T. 2005: Restoration ecology meets carabidology: effects of floodplain restitution on ground beetle (Coleoptera: Carabidae). - Biodivers. Conserv. 14: 1583-1606.

Harrell F.E. JR. 2016: Hmisc: Harrell Miscellaneous. R Package Version 3.17-4. URL: https://cran.r-project.org/web/packages/ Hmisc/index.html.

Heydemann B. 1957: Die Biotopstruktur als Raumwiderstand und Raumfülle für die Tierwelt. — Verh. Dtsch. Zool. Ges. 50: 332-347.

HLAVÁČ P. \& Magrini P. 2016: New species of Winklerites from eastern Serbia (Coleoptera: Carabidae: Trechinae). - Acta Entomol. Mus. Natl. Pragae 56: 71-78.

HoneK A. 1988: The effect of crop density and microclimate on pitfall catches of Carabidae, Staphylinidae (Coleoptera) and Lycosidae (Araneae) in cereal fields. - Pedobiologia 32: 233-242.

Horvatovich S. 1993: Liste der Carabiden-Arten (Coleoptera, Carabidae) Ungarns (Stand, 1991). — Janus Pannonius Múz. Évk. 37[1992]: 5-12.

Humphrey J.W., Hawes C., Peace A.J., Ferris-Kaan R. \& Jukes M.R. 1999: Relationships between insect diversity and habitat characteristics in plantation forests. - For. Ecol. Manag. 113: $11-21$

HưRKA K. 1992: Ground Beetles (Carabidae). Vol. 1. Academia, Prague, 196 pp. [in Czech].

HŮRKA K. 1996: Carabidae of the Czech and Slovak Republics. Kabourek, Zlín, 565 pp. [in Czech and English].

IgONDOVÁ E. \& MAJZLAN O. 2015: Assemblages of ground beetles (Carabidae, Coleoptera) in peatland habitat, surrounding dry pine forests and meadows. - Folia Oecol. 42: 21-28.

Іто M., IтоU K. \& Iто K. 2010: Are carabid beetles suitable biotic indicators of insecticide impact in potato fields? - Appl. Entomol. Zool. 45: 435-447.

IvaniČ Porhajašová J., Noskovič J. \& Babošová M. 2016: Coleoptera families fluctuation depending on the organic fertilizers application. - Acta Fytotechn. Zootechn. 19: 150-156.

Ivanič Porhajašová J., Petrovičová K., Mlyneková E., Ernst D., Babošová M., NoskoviČ J. \& KRUMPÁlová Z. 2019: Impact of soil management on biodiversity of epigeic groups. - Appl. Ecol. Env. Res. 17: 13897-13908.

Jacobs C.T., Scholtz C.H., Escobar F. \& Davis A.L.V. 2010: How might intensification of farming influence dung beetle diversity (Coleoptera: Scarabaeidae) in Maputo Special Reserve (Mozambique)? - J. Insect Conserv. 14: 389-399.

JANUSCHKE K. \& VERDONSCHOT R.C.M. 2016: Effects of river restoration on riparian ground beetles (Coleoptera: Carabidae) in Europe. - Hydrobiologia 769: 93-104.

JÁSZAY T. \& JÁSZAYOVÁ A. 2019: New interesting findings of beetles (Coleoptera: Bothrideridae, Carabidae, Derodontidae, Leiodidae, Melasidae, Staphylinidae, Tenebrionidae) in Slovakia. - Biodivers. Environ. 10: 25-37 [in Slovak, English abstract]. Jost L. 2006: Entropy and diversity. — Oikos 113: 363-375.

Kagawa Y. \& Maeto K. 2014: Ground beetle (Coleoptera: Carabidae) assemblages associated with a satoyama landscape in Japan: the effects of soil moisture, weed height, and distance from woodlands. - Appl. Entomol. Zool. 49: 429-436. 
Kaiser W., Avenant N.L. \& Haddad C.R. 2009: Assessing the ecological integrity of a grassland ecosystem: the applicability and rapidity of the SAGraSS method. - Afr. J. Ecol. 47: 308-317.

Kotze D.J., Brandmayr P., Casale A., Dauffy-Richard E., Dekoninck W., Koivula M.J., Lövei G.L., Mossakowski D., NoordiJK J., PaARmann W. et AL. 2011: Forty years of carabid beetle research in Europe - from taxonomy, biology, ecology and population studies to bioindication, habitat assessment and conservation. - ZooKeys 100: 55-148.

Kromp B. 1999: Carabid beetles in sustainable agriculture: a review on pest control efficiency, cultivation impacts and enhancement. - Agricult. Ecosyst. Environ. 74: 187-228.

Lapin M., Mikulová K. \& ŠŤAstnÝ P. 2019: The Current Climatic Characteristics in Bratislava Karlove Ves and Prediction of the Possible Changes in the Future for Years 2025, 2050, 2075. Slovak Hydrometeorological Institute, Bratislava, 41 pp. [in Slovak].

LIK M. 2010: The influence of habitat type on the population dynamics of ground beetles (Coleoptera: Carabidae) in marshland. - Ann. Soc. Entomol. Fr. 46: 425-438.

Litavský J., Stašiov S., Svitok M., Michalková E., Majzlan O., ŽARNOVIČAN H. \& FEDOR P. 2018: Epigean communities of harvestmen (Opiliones) in Pannonian Basin floodplain forests: an interaction with environmental parameters. - Biologia 73: 753-763.

Litavský J., Majzlan O., Stašıov S., Svitok M., Žarnovičan H. \& FEDOR P. 2021: Soil-dwelling communities of weevils (Coleoptera, Curculionoidea: Brentidae, Curculionidae) in Central European floodplain forests: a comparative interaction with environmental parameters. — Biologia 76: 179-192.

LiU R.T., Zhaо H.L. \& Zнао X.Y. 2013: Changes in soil macrofaunal community composition under selective afforestation in shifting sand lands in Horqin of Inner Mongolia, northern China. - Ecol. Res. 28: 1-8.

Lorenz W. 2019: CarabCat: global database of ground beetles (version Oct 2017). In Roskov Y., Ower G., Orrell T., Nicolson D., Bailly N., Kirk P.M., Bourgoin T., DeWalt R.E., Decock W., Nieukerken E. et al. (eds): Species 2000 \& ITIS Catalogue of Life, 2019 Annual Checklist. Naturalis, Leiden, URL: www. catalogueoflife.org/annual-checklist/2019.

Lövei G.L. \& SunderLand K.D. 1996: Ecology and behavior of ground beetles (Coleoptera: Carabidae). - Annu. Rev. Entomol. 41: 231-256.

Magura T., Tóthmérész B. \& Elek Z. 2003: Diversity and composition of carabids during a forestry cycle. - Biodivers. Conserv. 12: 73-85.

MAJZlan O. \& LitaVsKÝ J. 2015: Invertebrates (Evertebrata) - indicators of the landscape changes. - Entomofauna Carpathica 27: 63-68 [in Slovak, English abstract].

MaJZLAN O. \& LiTAVSKÝ J. 2017: Topical affinity of beetles (Coleoptera) to soil in floodplain forests along the Tisa and Begej rivers (Republic of Serbia). - Entomofauna Carpathica 29: 13-26 [in Slovak, English abstract].

Matalin A.V. 2003: Variations in flight ability with sex and age in ground beetles (Coleoptera, Carabidae) of south-western Moldova. - Pedobiologia 47: 311-319.

Migliorini M., Petrioli A. \& Bernini F. 2002: Comparative analysis of two edaphic zoocoenoses (oribatid mites and carabid beetles) in five habitats of the 'Pietraporciana' and 'Lucciolabella' Nature Reserves (Orcia Valley, central Italy). — Acta Oecol. 23: 361-374.

Müller-MotzFeld G. 2004: Adephaga 1. Carabidae (Laufkäfer) In Freude H., Harde K.W., Lohse G.A. \& Klausnitzer B. (eds):
Die Käfer Mitteleuropas. 2nd ed. Spektrum, Heidelberg, Berlin, pp. 1-521.

NAKLÁDAL O. 2008: Results of a faunistic survey of beetles (Coleoptera) in floodplain forests of the Litovelské Pomoraví Protected Landscape Area (Czech Republic, Northern Moravia) in 2006. - Klapalekiana 44: 237-269.

Negro M., Isaia M., Palestrini C., Schoenhofer A. \& Rolando A. 2010: The impact of high-altitude ski pistes on grounddwelling arthropods in the Alps. - Biodivers. Conserv. 19: 1853-1870.

Niemelä J. 2001: Carabid beetles (Coleoptera: Carabidae) and habitat fragmentation: a review. - Eur. J. Entomol. 98: 127132.

Niemelä J., Haila Y., Halme E., Lahti T., Pajunen T. \& Punttila P. 1988: The distribution of carabid beetles in fragments of old coniferous taiga and adjacent managed forest. - Ann. Zool. Fenn. 25: 107-119.

Niemelä J., Kotze J., Ashworth A., Brandmayr P., Desender K., New T., Penev L., Samways M. \& Spence J. 2000: The search for common anthropogenic impacts on biodiversity: a global network. - J. Insect Conserv. 4: 3-9.

Oksanen J., Blanchet F.G., Kindt R., Legendre P., Minchin P.R., O'Hara R.B., Simpson G.L., Solymos P., Stevens M.H.H. \& Wagner H. 2016: Vegan: Community Ecology Package. $R$ Package Version 2.3-4. URL: https://cran.r-project.org/web/ packages/vegan/index.html.

Paill W., Gunczy J. \& Hristovski S. 2018: The Vjosa-floodplains in Albania as natural habitat for ground beetles: a hotspot of rare and stenotopic species (Coleoptera: Carabidae). - Acta ZooBot Austria 155: 269-306.

Paoletti M.G., D’Inca A., Tonin E., Tonon S., Migliorini C., Petruzzelli G., Pezzarossa B., Gomiero T. \& Sommaggio D. 2010: Soil invertebrates as bio-indicators in a natural area converted from agricultural use: the case study of VallevecchiaLugugnana in north-eastern Italy. - J. Sustain. Agr. 34: 38-56.

Pavićević D., Vesović N., Popović M. \& ĆurČıć S. 2018: A new troglobitic ground beetle (Carabidae: Trechinae) from eastern Serbia. - Nature Conserv. 68: 61-66.

Pearce J.L., Venier L.A., McKee J., Pedlar J. \& McKenney D. 2003: Influence of habitat and microhabitat on carabid (Coleoptera: Carabidae) assemblages in four stand types. - Can. Entomol. 135: 337-357.

Pearson D.L. \& Cassola F. 2005: A quantitative analysis of species descriptions of tiger beetles (Coleoptera: Cicindelidae), from 1758 to 2004, and notes about related developments in biodiversity studies. - Coleopt. Bull. 59: 184-193.

Pearson D.L. \& Cassola F. 2007: Are we doomed to repeat history? A model of the past using tiger beetles (Coleoptera: Cicindelidae) and conservation biology to anticipate the future. $-J$. Insect Conserv. 11: 47-59.

Porhajašová J. \& ŠUstek Z. 2011: The Spatial Structure of Invertebrate Communities with a Focus on Family Carabidae in the Žitavský Luh Nature Reserve. Slovak University of Agriculture, Nitra, 77 pp. [in Slovak, English abstract].

Porhajašová J., ŠUstek Z., Noskovič J., URMinSKÁ J. \& OndRiší P. 2010: Spatial changes and succession of carabid communities (Coleoptera, Insecta) in seminatural wetland habitats of the Žitava river foodplain. - Folia Oecol. 37: 75-85.

Rainio J. \& Niemelä J. 2003: Ground beetles (Coleoptera: Carabidae) as bioindicators. - Biodivers. Conserv. 12: 487-506.

R Core Team 2016: $R$ : A Language and Environment for Statistical Computing. R Foundation for Statistical Computing, Vienna, URL: https://www.r-project.org. 
Republic Hydrometeorological Service of Serbia (Yearbooks) 1999-2010: URL: http://www.hidmet.gov.rs/ciril/meteorologija/klimatologija_godisnjaci.php [in Serbian].

Rothenbücher J. \& SCHAEFer M. 2006: Submersion tolerance in floodplain arthropod communities. - Basic Appl. Ecol. 7: 398-408.

Rushton S.P., Eyre M.D. \& LufF M.L. 1990: The effects of management on the occurrence of some ground beetle species in grassland. In Stork N.E. (ed.): The Role of Ground Beetles in Ecological and Environmental Studies. Intercept, Andover, pp. 209-216.

Schwerk A. 2008: Model of the Rate of Succession of Epigeic Carabid Beetles (Coleoptera: Carabidae) in Degraded Areas. Forest Research Institute, Sękocin Stary, 71 pp.

SkŁodowsKi J. 2014: Consequence of the transformation of a primeval forest into a managed forest for carabid beetles (Coleoptera: Carabidae) - a case study from Białowieża (Poland). - Eur. J. Entomol. 111: 639-648.

Song M.Y., Leprieur F., Thomas A., Lek-Ang S., Chon T.S. \& LEK S. 2009: Impact of agricultural land use on aquatic insect assemblages in the Garonne river catchment (SW France). Aquat. Ecol. 43: 999-1009.

Spellerberg I.F. \& Fedor P.J. 2003: A tribute to Claude Shannon (1916-2001) and a plea for more rigorous use of species richness, species diversity and the 'Shannon-Weiner' index. Global Ecol. Biogeogr. 12: 177-179.

StaŠIOv S. 2015: Ecology of Soil Organisms (Soil Animals). Technical University in Zvolen, Zvolen, 150 pp. [in Slovak].

ŠEJNOHOVÁ H. 2006: The dynamics of carabid beetles (Carabidae) of floodplain forest in Southern Moravia. - Acta Univ. Agric. Silvic. Mendel. Brun. 54: 107-120.

ŠTEFÁNIK M. \& FedOR P. 2020: Environmental stress in Parnassius apollo reflected through wing geometric morphometrics in a historical collection with a possible connection to habitat degradation. - Nat. Conserv. 38: 79-99.

ŠUSTEK Z. 1994a: Classification of the carabid assemblages in the floodplain forests in Moravia and Slovakia. In Desender K., Dufrêne M., Loreau M., Luff M.L. \& Maelfait J.P. (eds): Carabid Beetles: Ecology and Evolution. Series Entomologica, Vol. 51. Springer, Dordrecht, pp. 371-376.

ŚUSTEK Z. 1994b: Floodplain forests and the fate of their fauna. Vesmir 73: 326-329 [in Slovak].

ŠUSTEK Z. 1994c: Impact of water management on a carabid community (Insecta, Coleoptera) in a Central European floodplain forest. — Quad. Staz. Ecol. Civ. Mus. St. Nat. Ferrara 6: 293313.

ŠUSTEK Z. 2000: Carabid communities and their application as a supplementary characteristic of the geobiocoenological units: problems and state of the knowledge. In Štykar J. \& Čermák P. (eds): Geobiocoenological Typification of the Landscape and its Application. Faculty of Forestry and Wood Technology, Mendel University, Brno, pp. 18-30 [in Slovak].

ŠUSTEK Z. 2004a: Characteristics of humidity requirements and relation to vegetation cover of selected Central-European carabids (Col., Carabidae). In Polehla P. (ed.): Proceedings of the International Conference "Evaluation of the State and Development of Forest Geobiocenoses". Faculty of Forestry and Wood Technology, Mendel University, Brno, pp. 210-214 [in Slovak, English abstract].

ŠUSTEK Z. 2004b: Transport of water, chemicals and energy in the system soil-crop canopy-atmosphere. In Kosorin K., Novák V., Štekauerová V., Šútor J. \& Túnyi I. (eds): Changes in Car- abid (Coleoptera, Carabidae) Communities along a Moisture

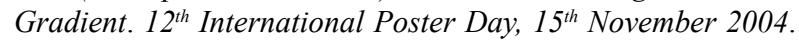
Bratislava, pp. 456-462.

Šustek Z. 2005: Ground beetles (Coleoptera, Carabidae) and their response to changes of vegetation in alpine ecosystems. - Folia Faun. Slov. 10: 19-22 [in Slovak, English abstract].

ŠUsteK Z. 2010: Succession of carabid communities in different types of reed stands in Central Europe. - Oltenia, Studii şi comunicări, Științele Naturii 26: 127-138.

ŠUSTeK Z. 2012: Ground beetles (Coleoptera: Carabidae). In Holecová M., Christophoryová J., Mrva M., Roháčová M., Stašiov S., Štrichelová J., Šustek Z., Tirjaková E., Tuf H.E., Vd’ačný P. \& Zlinská J. (eds): Biodiversity of Soil Micro- and Macrofauna in Oak-Hornbeam Forest Ecosystem on the Territory of Bratislava. Comenius University, Bratislava, pp. 73-94.

Tallósi B. \& Sekulić R. 1989: The Carabidae (Coleoptera) fauna on the sodic soils of the Tisa Valley (Yugoslavia). - Tiscia (Szeged) 23: 87-95.

Thomas C.F.G., Brown N.J. \& Kendall D.A. 2006: Carabid movement and vegetation density: implications for interpreting pitfall trap data from split-field trials. - Agric. Ecosyst. Environ. 113: 51-61.

Trautner J. \& Geigenmüller K. 1987: Tiger Beetles, Ground Beetles: Illustrated Key to the Cicindelidae and Carabidae of Europe. Josef Margraf, Aichtal, 487 pp.

Tuomisto H. 2012: An updated consumer's guide to evenness and related indices. - Oikos 121: 1203-1218.

VÁsquez-Vélez L.M., Bermúdez C., Chacón P. \& Lozano-ZamBRANO F.H. 2010: Analysis of the richness of Staphylinidae (Coleoptera) on different scales of a sub-Andean rural landscape in Colombia. - Biodivers. Conserv. 19: 1917-1931.

VehviläInen H., Koricheva J. \& RUOHOMÄKI K. 2008: Effects of stand tree species composition and diversity on abundance of predatory arthropods. - Oikos 117: 935-943.

VENN S. 2003: Urbanization effects on carabid diversity in boreal forests. - Eur. J. Entomol. 100: 73-80.

Vician V., Svitok M., Michalková E., Lukáčik I. \& Stašiov S. 2018: Influence of tree species and soil properties on ground beetle (Coleoptera: Carabidae) communities. - Acta Oecol. 91: $120-126$.

Wiтtich W. 1942: Die Aktivierung von Rohhumus extrem ungünstiger Beschaffenheit. - Z. Forst- und Jagdwesen 74: 241-271.

Wiтtich W. 1943: Untersuchungen über den Verlauf der Streuzersetzung auf einem Boden mit Mullzustand II. - Forstarchiv 19: $1-18$.

ZaHRADNí P. 2017: Check-List of Beetles (Coleoptera) of the Czech Republic and Slovakia. Lesnická práce, Kostelec nad Černými lesy, 544 pp. [in Czech and English].

Zou Y., Sang W., Bai F. \& Axmacher J.C. 2013: Relationships between plant diversity and the abundance and $\alpha$-diversity of predatory ground beetles (Coleoptera: Carabidae) in a mature Asian temperate forest ecosystem. - PLoS ONE 8: e82792, $7 \mathrm{pp}$.

Zvaríková M., Kiselák J., Zvarík M., Masarovič R., Prokop P. \& FEDOR P. 2016: Ecological gradients and environmental impact in the forest dwelling Haplothrips subtilissimus (Thysanoptera: Phlaeothripidae) phenotypic variability. - Ecol. Indic. 66: $313-320$.

Received September 30, 2020; revised and accepted December 10, 2020 Published online January 22, 2021 